\title{
Pulmonary Function Testing in Office Practice
}

\author{
Soumya Swaminathan \\ Tuberculosis Research Centre, Indian Council of Medical Research Chetput, Chennai
}

\begin{abstract}
With the wide availability of simple to operate pulmonary function equipment, these tests can now be performed in the office or clinic setting. The use of pulmonary function tests in the assessment of patients with pulmonary diseases has thus expanded. These simple tests rely on data generated from a forced expiratory vital capacity maneuver which can be performed reliably by most children over the age of 6 or 7 years. Pulmonary function tests provide both diagnostic and prognostic information and help in the management of a number of respiratory diseases. The number of tests available vary with the level of sophistication of the pulmonary function laboratory; in most situations simple spirometry is adequate. Several new and inexpensive peak flow meters are now available for home use; these are very useful for home monitoring of asthma patients. Physicians caring for patients with asthma or other chronic respiratory diseases should be encouraged to use these tests routinely in the management of these children. (Indian J Pediatr 1999; 66 : 905-914)
\end{abstract}

Key words : Pulmonary function tests; Spirometry; Asthma.

Pulmonary function tests (PFTS) are an essential part of the investigation of many respiratory diseases. Though they do not provide a specific diagnosis, they help us to understand the physiologic and functional abnormalities and to follow the course of the disease. Asthma is one of the commonest problems in childhood and its diagnosis and management pose a major problem to the clinician. Just as estimation of blood sugar is an integral part of management of diabetes mellitus pulmonary function tests provide an objective measure of pulmonary disability and should be used routinely in the management of children with chronic respiratory diseases. Due

Reprint requests : Soumya Swaminathan, MD, MNAMS, Deputy Director, Tuberculosis Research Centre, Indian Council of Medical Research, Chetput, Chennai - 600031; Fax : (044) 8262137 to the technical difficulties involved, pulmonary function tests are not done routinely in children below the age of 6 to 7 years. However, the introduction of simple to operate pulmonary function equipment now allows these studies to be done in older children in the general outpatient setting.

Simple spirometry is good enough in most situations, as more sophisticated equipment may not be widely available. Additional information can be obtained in special situations from further tests including measurement of lung volumes and capacities, diffusion capacity, respiratory muscle strength measurement and exercise testing. It is imperative to have normal established values for each parameter for the population. A number of variables like ethnic origin, age, sex, height and body surface area can affect the pulmonary function parameters. Ideally each laboratory should 
have the norms for the population it serves.

\section{Selection of Pulmonary Function Tests}

Unless a specific physiologic abnormality has al ready been documented, the patient is studied to characterize ${ }^{1}$ :

1. Lung volumes : By body plethysmography or gas dilution technique.

2. Lung mechanics: Flow rates by spirometry and airway resistance by plethysmography.

3. Distribution of ventilation : Single breath or multiple breath nitrogen washout,

4. Gas exchange : Blood gas analysis or diffusing capacity of the lung.

Further studies such as provocation tests (aerosol bronchodilators, histamine, methacholine or exercise), ventilatory response to exercise or sleep studies may also be indicated, depending on the type of respiratory disorder that is suspected.

The major clinical indications for performing PFTs are as follows:

1. To determine if symptoms and signs such as dyspnoea, cough and cyanosis are of respiratory origin

2. To characterize pulmonary diseases physiologically. Although PFTs are not diagnostic for a specific pulmonary disorder, they may suggest disease etiology.

3. To monitor the course of lung function impairment. PFTs often provide more sensitive, objective and quantitative information concerning changes in lung function than patient history and physical examination.
4. To determine the effectiveness of therapy e.g. aerosol bronchodilator treatment in asthma and steroids in interstitial lung diseases.

5. To assist in the preoperative planning of general anesthesia and in anticipating the need for postoperative oxygen and/ or assisted ventilation. Preoperative pulmonary function evaluation is particularly important in patients with chest wall deformities e.g. scoliosis, collagen vascular diseases and neuromuscular diseases.

\section{Subdivision of Lung Volumes}

There are 4 lung volumes and 4 capacities represented diagrammatically in fig. $1^{2}$. Each lung capacity is the sum of two or more lung volumes. Tidal volume (TV) is the amount of gas exchanged with normal, quiet breathing. Inspiratory reserve volume (IRV) is the additional gas that can be inspired after the end of a quiet inspiration and expiratory reserve volume (ERV) is the amount of gas that can be expired from the resting end expiratory level fully up to residual volume. Residual volume (RV) is the volume of air left in the lung at the end of a maximal expiration and together with ERV equals functional residual capacity (FRC). Inspiratory capacity (IC) is the amount of gas that can be inspired from the end of a quiet expiration and is the sum of TV and IRV. Vital capacity (VC) is the sum of TV, IRV and ERV and is the amount that can be expired after a maximal inspiration. Total lung capacity (TLC) which is the sum of all four lung volumes is the amount of gas in fully inflated lungs. These subdivisions show changes in different lung diseases that help us to understand the nature of the 


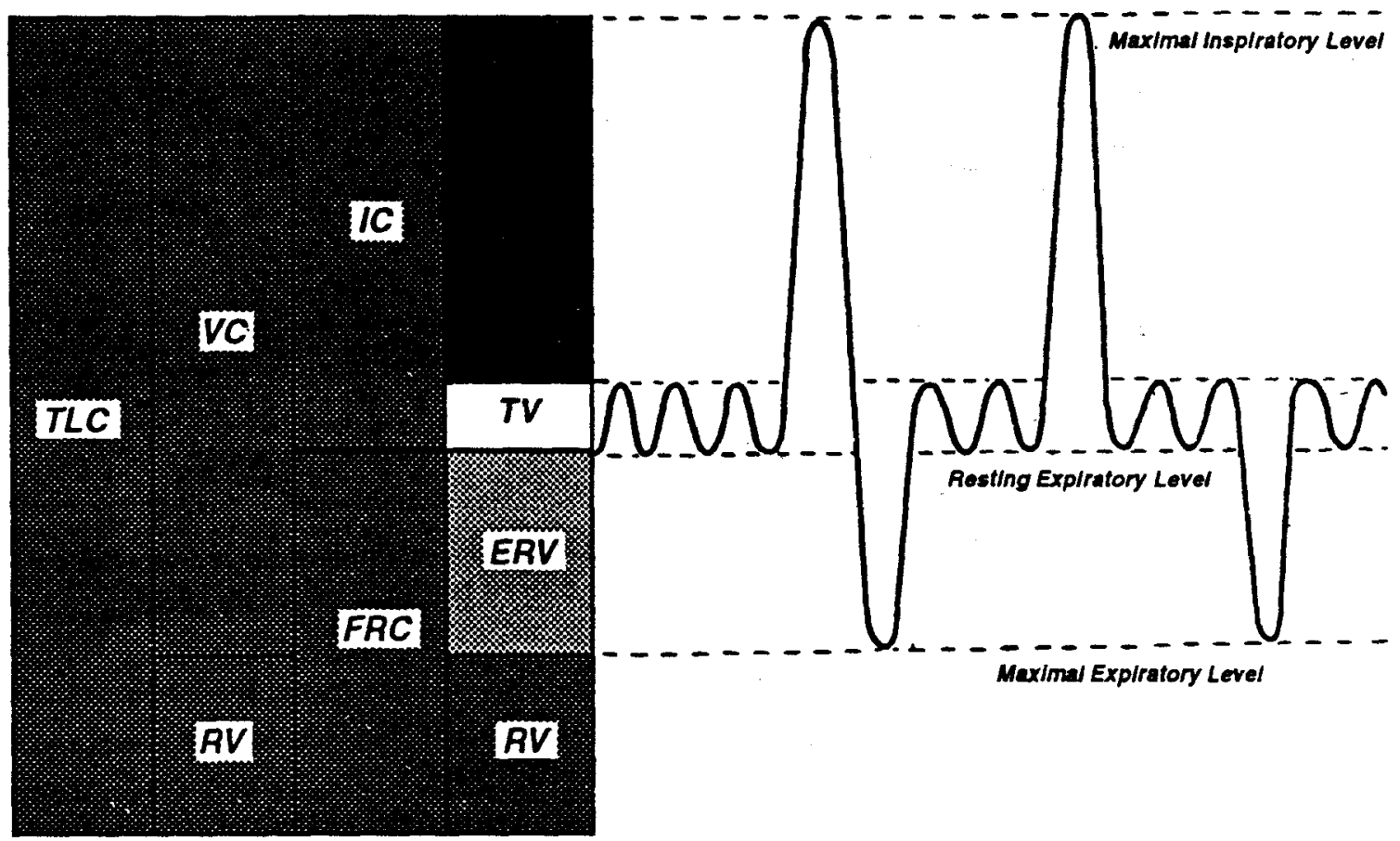

Fig. 1. Lung volumes as they appear on a spirographic tracing. The four primary lung volumes and the four lung capacities are shown.

defect.

\section{Flow Rates}

The rate at which the volume of gas is exhaled is flow and it is recorded as L/ min or $\mathrm{L} / \mathrm{sec}$. The peak expiratory flow rate (PEFR) occurs early in expiration and is dependent on patient effort and large airways resistance. Resistance of the smaller airways has a greater effect on flow at lower lung volumes. To study flow at lower lung volumes, flow is averaged over the mid portion of the lung volume $\left(\mathrm{FEF}_{25 \%-75 \%}\right)$. Similarly, flow rates can be determined at different lung volumes (from the flow volume curve) e.g. Vmax at $80 \%$ TLC indicates the maximum flow at $80 \%$ of TLC or Vmax $50 \%$ VC indicates flow rate at 50\% vital capacity. These flow rates are more useful in small airway diseases.
Spirometry measures the volume of air exhaled from the lungs during a maximal expiratory maneuver (Fig. 2). The forced vital capacity is the total volume of air that can be exhaled after a full inspiration. Though it is measured by spirometry it is technically a volume and not a flow rate. Forced expiration is begun at TLC and ends at RV and usually takes less than 3 seconds. Forced expiratory volume in 1 second $\left(\mathrm{FEV}_{1}\right)$ is the volume of air forcefully expired from full inflation in the first second. Both FVC and $\mathrm{FEV}_{1}$ are recorded in litres. Healthy children are able to exhale $>$ $80 \%$ of their FVC in 1 second. There is a trend for the $\mathrm{FEV}_{1} / \mathrm{FVC}$ ratio to decrease slightly after early adulthood. Since children younger than 7 years may not inspire to TLC or exhale to RV, valuable information concerning airway function in this age group can be obtained by a partial 

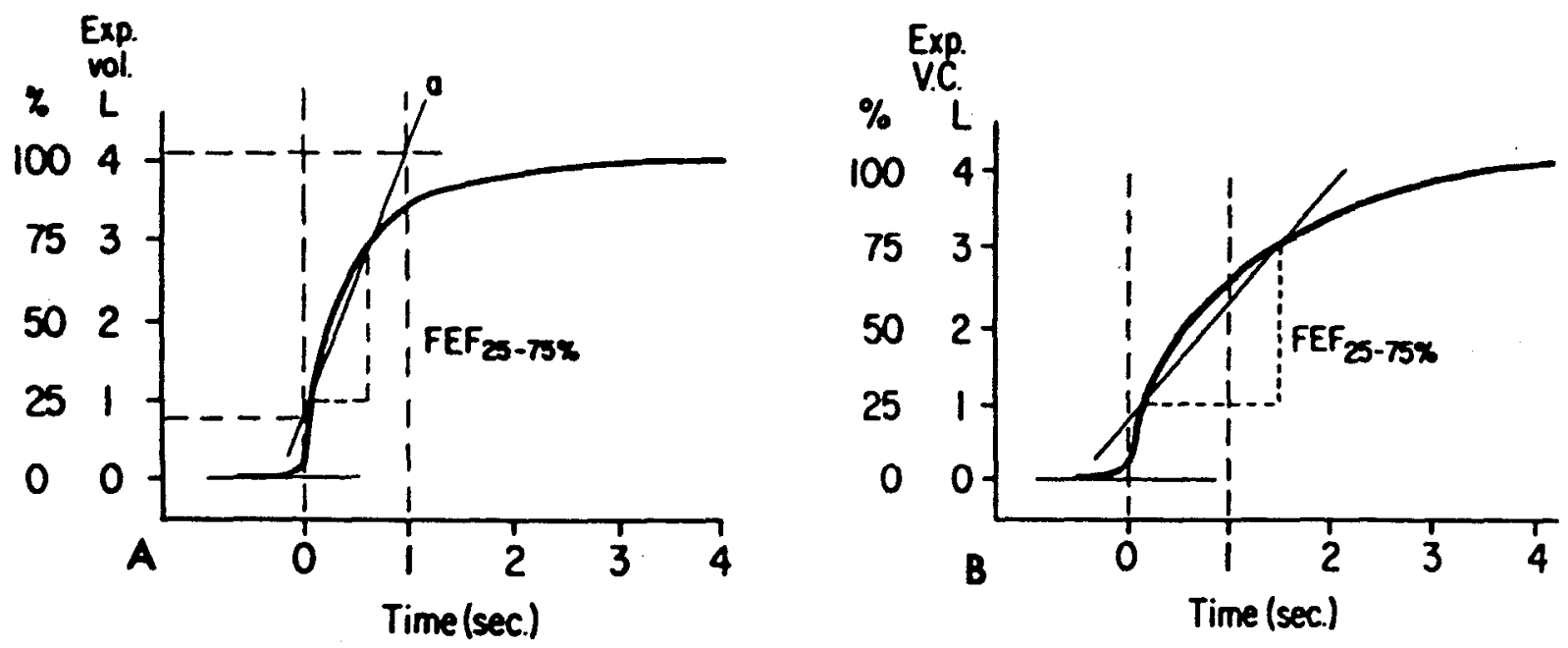

Fig. 2. Volume - time curves for a normal child (A) and a child with obstructive airways disease (B). The forced expiratory flow over the middle half of the vital capacity $\left(\mathrm{FEF}_{25 \%-75 \% \text {.) }}\right.$ can be calculated from this tracing.

'flow volume curve' measuring maximal expiratory flow at FRC (Vmax FRC).

Minimum specifications for spirometers for clinic and office use were proposed by the American Thoracic Society (1979) and updated in 1987. Any spirometer must calculate or display the FVC, FEV 1 and PEFR These pulmonary function tests are the most reproducible of all spirometric measurements, useful for longitudinal studies and. easiest to perform. Healthy children and adolescents aged 7 to 16 years perform pulmonary function studies as reproducibly as healthy adults ${ }^{5}$.

\section{The Testing Environment}

In order to achieve reasonable success in obtaining PFTs in children, factors that are usually not important in testing the adult patient must be considered here. These include technician capability, the equipment and the laboratory facilities. Children need an honest, reassuring introduction to the lab and to the tests that they will be performing. The mouthpiece and nose clip should be given to the child and he/ she should have enough time getting used to these. Most importantly, the person administering the test should establish a good rapport with the child and gain his confidence. The child should be sitting erect throughout the test. The vital capacity should be reported as the largest volume obtained from any of the respiratory maneuvers. Older children and adolescents should have the best of the 3 tests taken as the "truest" measure of lung function while younger children may require more than 3 tests $^{6}$. The "best" test is the one with the greatest sum of FVC and FEV .

Interpreting Spirometry : Spirometry not only allows the characterization of a patient's lung function against reference values but also defines the disease dass. Most lung diseases can be classified as obstructive, restrictive or mixed - type processes. 

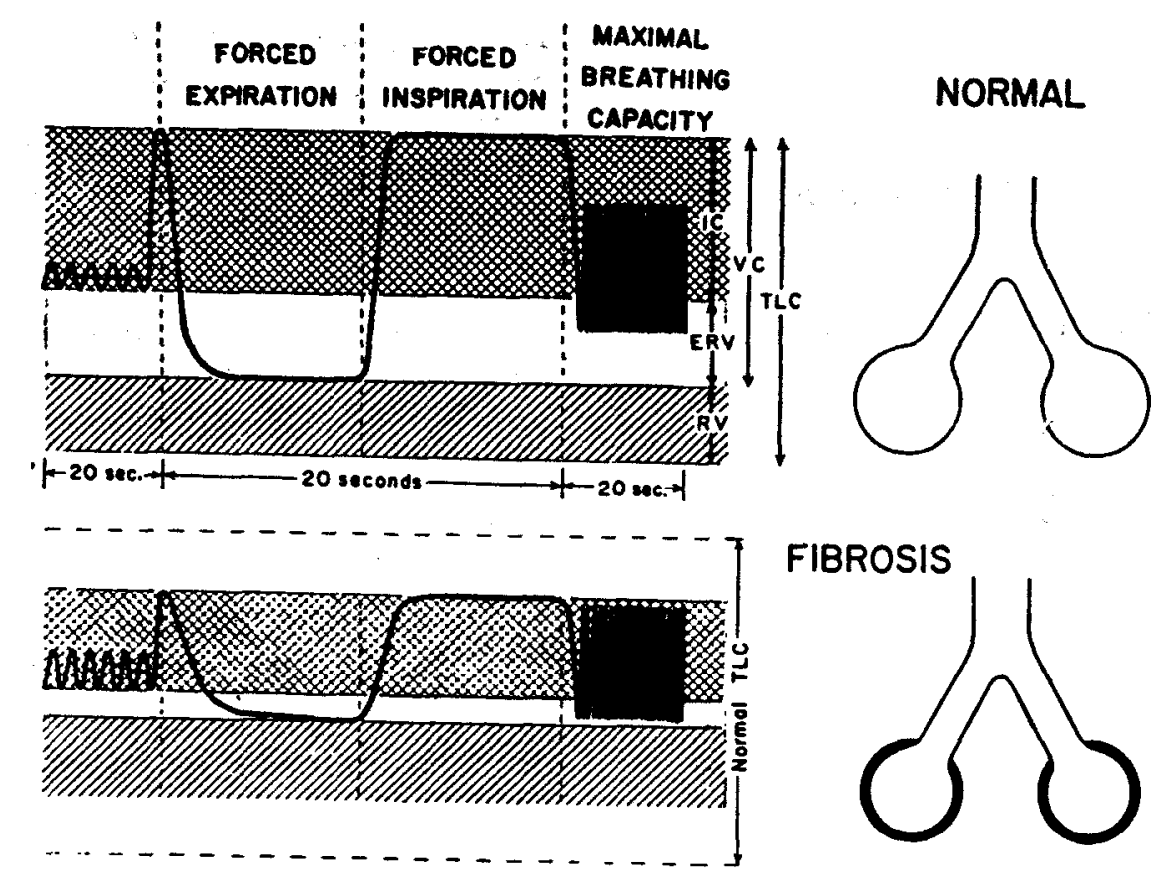

FIBROSIS
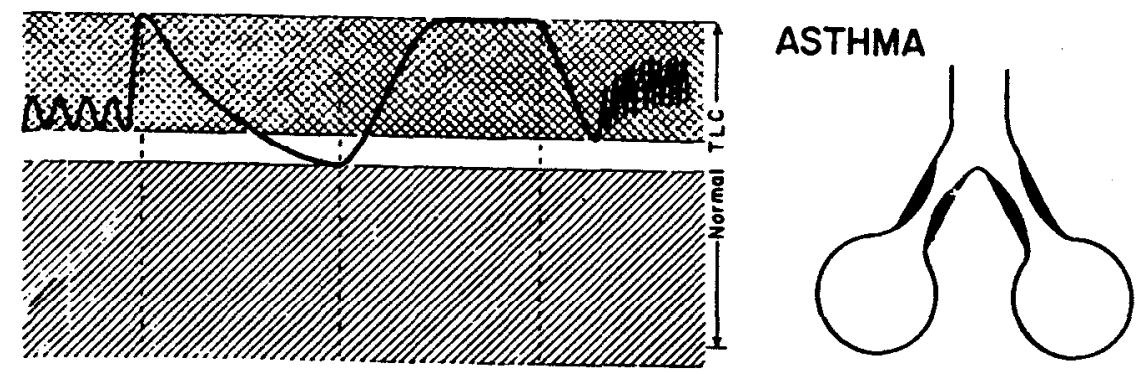

Fig. 3. The spirogram is shown in relation to total lung capacity, vital capacity, functional residual capacity, residual volume, expiratory reserve volume and inspiratory capacity of each patient. "Fibrosis" is an example of restrictive type of lung disease.

The VC is decreased in both obstructive and restrictive disease but while the RV is increased due to gas trapping in obstructive disease resulting in an increased RV/ TLC ratio, the RV, FRC and TLC are all proportionately reduced in restrictive disease (Fig 3). Since the flow rates are not affected in most restrictive lung disorders, the $\mathrm{FEV}_{1} / \mathrm{FVC}$ ratio will be normal but this is reduced in obstructive diseases. Thus the $\mathrm{FEV}_{1}$ / FVC ratio usually allows disease classification without the need to measure lung volumes if the facilities do not exist (Table 1).

The configuration of the flow-volume and volume-time curves when taken from a maximal forced expiration can provide valuable information about the disease class when compared with the normal curve (Fig. 4). In obstructive diseases, flow decreases rapidly as gas is exhaled giving a flow volume curve which is convex towards the volume axis. In restrictive disease, the curve shape is normal but smaller 
TABle 1. Abnormal Spirometric Patterns

1. Obstructive Pattern

N ormal or decreased VC

Decreased maximal expiratory flow rates

$\mathrm{FEV}_{1} / \mathrm{FVC}$ below $80 \%$

Decreased MVV

Increased RV and RV/ TLC ratio

Increased airway resistance

2. Restrictive Pattern

Decreased VC and TLC

$\mathrm{FEV}_{1}$ decreased

Relatively normal forced expiratory flow rates

$\mathrm{FEV}_{1} / \mathrm{FVC}$ ratio normal or increased

Normal or decreased MW (late stage)

Decreased RV and normal RV/ TLC ratio

3. M ixed or Indeterminate Pattern

Decreased VC

Decreased expiratory flow rates

Decreased MVV

VC : Vital Capacity

FEV $\quad$ : Forced Expiratory Volume (in 1 set)

FVC : Forced Vital Capacity

MVV : Maximal Voluntary Ventilation

RV : Residual Volume

TLC : Total Lung Capacity

than the normal curve

Spirometric data interpretation should include an assessment of the quality of the study. Certain criteria have been laid down for an acceptable test which include:

(a) Appropriate curve shape which is artifact free

(b) Sustained expiration for at least 3 seconds

(c) At least 3 forced vital capacities within $10 \%$ of the best effort and

(d) Satisfactory effort by the patient as observed by the tester.

Problems are usually due to inadequate patient effort or coughing and can be cor- rected by additional instruction, incentives or allowing the patient to rest.

Forced expiratory flow at $25 \%$ to $75 \%$ of FVC $\left(\mathrm{FEF}_{25 \%}-75 \%\right)$ is a more sensitive indication of mild small airways obstruction than $\mathrm{FEV}_{1}$. Its disadvantage lies in a wide range of normal and also that the value can change depending on the lung volume at which it is measured.

\section{Response to Bronchodilators}

The diagnosis of reversible airway obstruction can be made by repeating the PFTs after administering an aerosolized bronchodilator to the patient. An increase in $\mathrm{FEV}_{1}$ of more than $15 \%$ would indicate the presence of reversible airways obstruction. If the baseline PFTs are normal and clinically there is a suspicion of hyperreactive airways disease, then a challenge with histamine, methacholine or exercise may be done in order to induce air way obstruction. In this case, a reduction in flow rates following the challenge would point to a diagnosis of obstructive airway disease.

\section{Peak Flow Meter}

It is a simple, portable and inexpensive device that measures peak expiratory flow rate in L/ min or L/ set. Because of its wide variation and effort dependence, it is more useful for monitoring progress of asthma than for diagnosis ${ }^{7}$. Fall in PEFR may be an early warning of an impending asthma attack, hence home monitoring of PEFR is very useful for patients. Patients can be asked to maintain a record of their PEFR 2 or 3 times a day and increase the medication if there is a fall $>20 \%$. Peak flow meters should also be available in the pediatrician's clinic so that the child's progress 


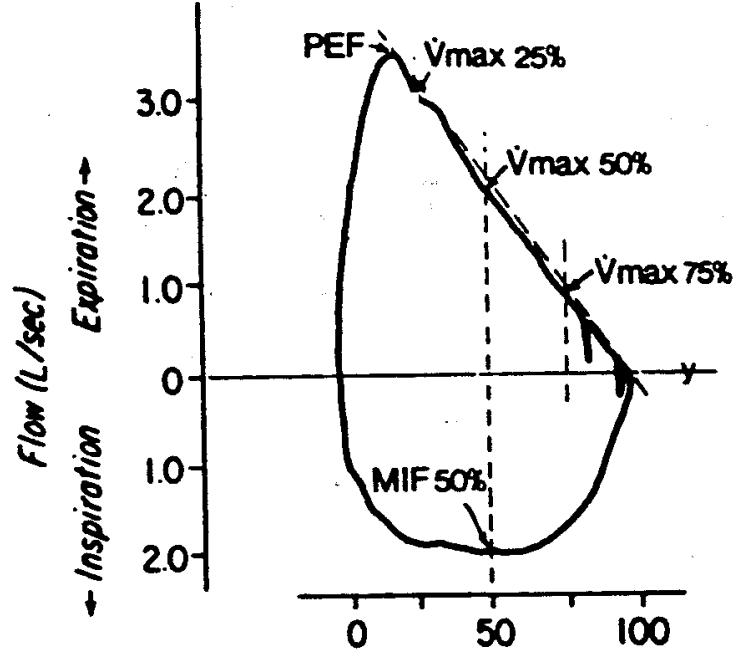

A

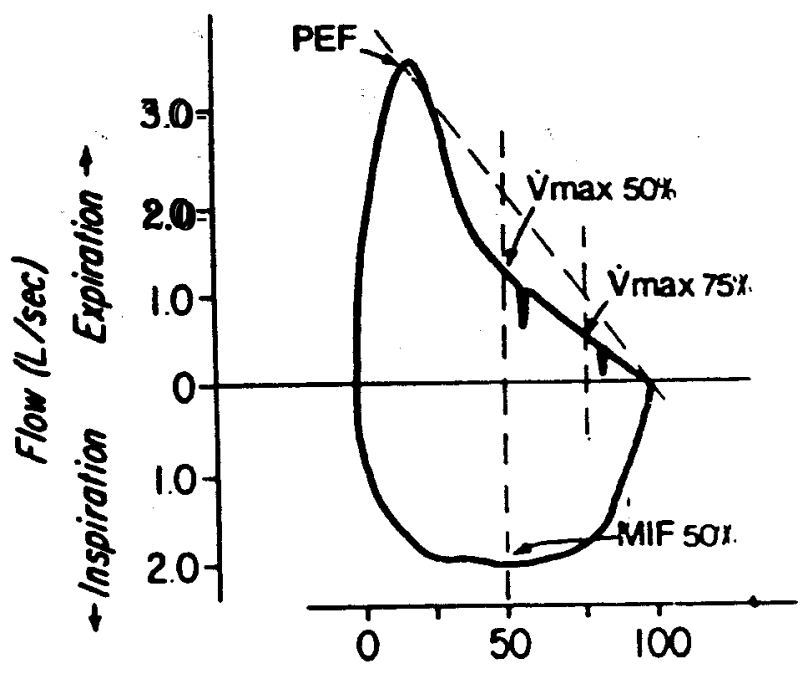

B

Expired vitol copacity $(\%)$

Fig. 4. Flow volume curves for a normal child $(A)$ and a child with moderate obstructive airways disease (B).

and asthma control can be objectively measured.

\section{Lung Volume Measurement}

Direct measurement of lung volumes is usually made either by body plethysmorgaphy or by gas dilution techniques. These methods are used to determine absolute lung volume, usually at FRC. Body plethysmography is preferable both for procedural and technical reasons but the instrument may not be available everywhere. With the rapid electrically activated mouth shutter used in plethysmography, it is possible to obtain the measurement of thoracic gas volume (TGV) which is the same as FRC. It is also possible to repeat this measurement until a consistent minimal value for each patient is determined. The calculation of lung volume by this technique is based on Boyle's law which states that the product of pressure and volume of gas remain constant, in a closed space at a fixed temperature. It is also possible to perform spirometry in the body plethysmograph itself, either using a pneumotachygraph or by connecting the mouthpiece to a spirometer. If the child is afraid of being confined alone, the study can be performed with the child seated on an adult's lap. Hence, both lung volumes and flow rates can be measured using a body plethysmograph.

Estimation of FRC by the helium dilution method is less reliable in the younger child because it requires more cooperation and does not measure poorly ventilated or non-ventilated areas of the lung. However, this method is relatively inexpensive and useful in children without obstructive airway disease. If the TGV measured by plethysmography exceeds the FRC $(\mathrm{He})$ by more than $400 \mathrm{ml}$, it indicates significant gas trapping. Once the patient's FRC (or TGV) and vital capacity is known, the residual volume (RV) can be calculated. RV is one of the most variable measurements of 


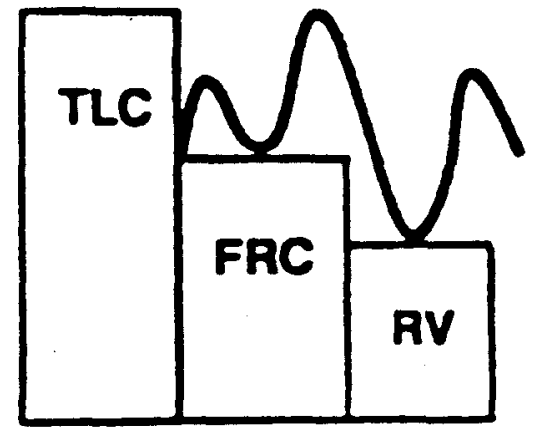

OBSTRUCTION

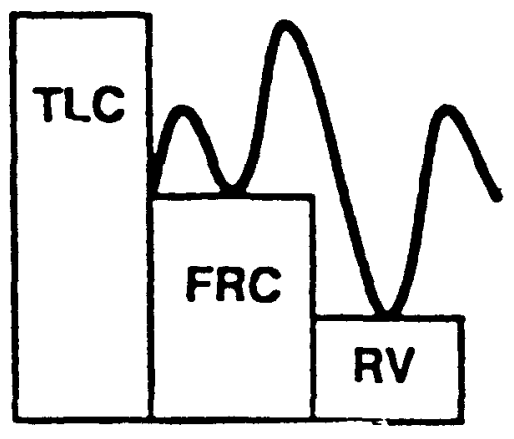

NOAMAL

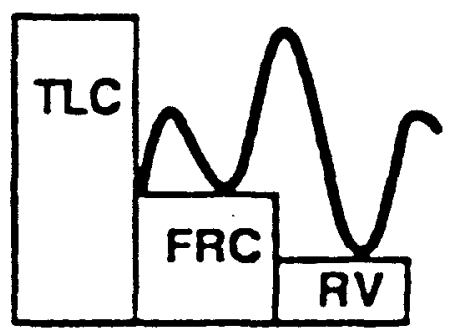

RESTRICTIVE

Fig. 5. Changes in lung volumes in different types of lung diseases.

lung function in children and must be interpreted with caution. RV cannot be measured by a spirometer, only calculated indirectly which may not be accurate.

Measuring lung volume allows determination of the RV/ TLC ratio which increases with gas trapping from obstructive disease. Lung volume measurement also helps to distinguish between obstructive and restrictive disease (Fig 5). With restrictive processes such as interstitial lung diseases, neuromuscular diseases or scoliosis the FRC, TLC and RV should be followed to assess the progression of illness, which decreases these values. In a child who is to undergo a major thoracic or abdominal surgery the inspiratory capacity might fall as much as $50-70 \%$ post-operatively due to pain and sedation. If the preoperative IC is $<30 \mathrm{ml} / \mathrm{kg}$ the child may need assisted ventilation following surgery.

\section{Airway Resistance}

Airway resistance (Raw) in older children is usually assessed using the body plethysmograph. Almost half the total airway resistance in children may be from the upper airway so direct measurement of R aw may not clearly represent resistance in the pulmonary airways. Resistance is usually converted to its reciprocal, airway conductance ( $\mathrm{Gaw}$ ) because this value is linearly related to lung volume. Conductance can then be normalized for increases in lung volume with growth by dividing it by FRC (specific airway conductance, SG aw). SG aw changes little during childhood in normals. Airway resistance is measured be cause spirometry only assesses the combined interaction of lung recoil and airway resistance and cannot distinguish which of these has resulted in a given change in lung function. However, in most clinical situations, children can be managed without measurement of Raw as decreased flow is rarely due to abnormal lung recoil.

\section{Distribution of Ventilation}

(a) Single breath : The most sensitive test of the uniformity of ventilation is the slope of phase III of the single breath $\mathrm{N}_{2}$ wash out curve. When elevated above $2.5 \% \mathrm{~N}_{2} / \mathrm{L}$, it indicates non-uniform distribution of ventilation. The closing volume or phase, IV of the curve reflects the elastic properties of the lung and is elevated in small airway disease. 
(b) Multiple breath : Forty breath nitrogen washout curve is more difficult to perform. If elevated it also indicates non uniform distribution of ventilation and can provide data regarding the size of the poorly ventilated lung compartment.

\section{Gas Exchange}

(a) Blood gas analysis : Arterial blood gas analysis provides the most sensitive index of lung function [oxygen $(0$,$) up-$ take and alveolar ventilation] in infants and children. The arterial $\mathrm{O}_{2}$ tension is generally the most sensitive index of lung disease and is most likely to be abnormal in patients with apparently minimal lung disease. The arterial $\mathrm{CO}$, tension reflects the adequacy of alveoIar ventilation. The radial and temporal arteries are the most accessible superficial arteries in newborn infants whereas the radial artery is often most accessible in children. The Allen test to determine ulnar artery patency should be performed prior to radial artery puncture. Temporal artery puncture should be avoided and placement of an indwelling temporal artery catheter has been associated with focal brain necrosis.

(b) Noninvasive : Although the diffusion capacity of the lung (D LCo) was developed to assess pulmonary membrane gas transport, it is actually a test of pulmonary capillary lung volume. The $D L c o$ is reduced in restrictive lung disorders. But when normalized for patients' alveolar volume (VA) the test may be normal. The DLco single breath requires optimal patient cooperation and a minimum exhaled gas volume of one litre so it is not possible in young children.

\section{Reference Values}

Lung volumes and flow rates vary with age, sex and ethnic group. Lung volumes in adult Indian patients have been shown to be $15-20 \%$ lower than Caucasian values*. In children it is particularly important to have age and sex matched reference values from a control population ${ }^{9-12}$. It is advisable for each lab to develop its own normal values using its own equipment or to use data generated from the same population. Regression equations to calculate lung function values are available for Caucasian and Indian adults and children.

When a patient's performance is evaluated against reference values from a similar population, it is called referenced testing. A patient's performance when tested against his or her own past performance is referred to as longitudinal testing. This is particularly valuable when observing a positive response to treatment or confirming progression of disease.

\section{REFEREN CES}

1. Platzer ACG and Keens TG. Pulmonary function testing in pediatric patients. In : Wilson AF (ed). Pulmonary Function Testing : Indications and Interpretation. London, Grune and Stratton, 1985; 275-292.

2. Forster RE, Dubois $A B$, Briscoe WA and Fisher AB. The Lung : Physiologic Basis of Pulmonary Function Tests. Chicago Yearbook Medical Publishers 1986.

3. Pfaff JK and Morgan WJ. Pulmonary function in infants and children. Pediatr Clin N A mer 1994; 40 : 401-423.

4. American Thoracic Society : Standardiza- 
tion of spirometry - 1987 update. A m Rev Respir Dis 1987; 136 : 1285-1298.

5. Nickerson B, Lemen R, Gerdes $C$ et al. Within-subject variability and percent change for significance of spirometry in normal subjects and in patients with cystic fibrosis. Am Rev Respir Dis 1980; $122: 859$.

6. Polgar G, Promadhat V (eds). Pulmonary Function Testing in Children : Techniques and Standards. Philadelphia, WB Saunders Co. 1971; 42-86.

7. Swaminathan $S$, Venkatesan $P$ and Mukundan R. Peak expiratory flow rate in South Indian children. Indian Pediatr 1993; 30 : 207-211.
8. Vijayan VK, Kuppurao KV, Venkatesan P, Sankaran K and Prabhakar R. Pulmonary function in healthy young adult Indians in Madras. Thorax 1990; 45 : 611-615.

9. Amdekar YK and Ugra D. Pulmonary function tests. Indian ] Pediatr 1996; 63 : 149-152.

10. Kulpati DDS and Talwar D. Pediatric pulmonary function testing. Indian Pediatr 1992; 29 :277-282.

11. Malik S and Jindal SK. Pulmonary function tests in healthy children. Indian Pediatr 1985; 22 : 677-681.

12. Chowgule RV, Shetye VM and Parmer JR. Lung function tests in normal Indian children. Indian Pediatr 1995; 32 : 181-19.

\section{EVALUATION OF DIPSTICK ASSAY FOR DIAGNOSIS OF P. FALCIPARUM MALARIA}

Rapid, accurate and affordable methods are needed for the diagnosis of malaria. Reported here is an evaluation of a new immunochromatographic strip. the PATH Falciparum malaria IC strip, which is impregnated with an immobilized IgM monoclonal antibody that binds to the HRP-II antigen of Plasmodium falciparum. In contrast to other commercially available kits marketed for rapid diagnosis of falciparum malaria, this kit is affordable in the malariaendemic world. Using microscopy and polymerase chain reaction (PCR) based methods as reference standards, two versions of the PATH test were compared for the detection of $P$. falciparum infection in 200 febrile travelers. As determined by PCR and microscopy, 148 travelers had malaria, 30 of whom $(33.8 \%)$ were infected with $P$. falciparum. Compared with PCR, the two versions of the path test hat initial sensitivities of $90 \%$ and $88 \%$ and specificities of $97 \%$ and $96 \%$, respectively, for the detection of falciparum malaria. When discrepant samples were retested blindly with a modified procedure (increased sample volume and longer washing step) the sensitivity and specificity of both kits improved to $96 \%$ and $99 \%$, respectively. The two remaining false negatives occurred in samples with $<100$ parasites per $\mu \mathrm{l}$ of blood. The accuracy, simplicity and predicted low cost may make this test a useful diagnostic tool in malaria-endemic areas.,

Abstracted from : Bull WHO 1999; Vol. 77, No. 7 : pp. 553 